\title{
PROJECTIONS ONTO SOME FUNCTION SPACES ${ }^{1}$
}

\section{B. GRÜNBAUM}

1. Introduction. The present note deals with estimates for norms of projections onto subspaces (of a special kind) of the Banach spaces of all bounded functions defined on infinite sets. The results obtained generalize those of Sobczyk [8] and McWilliams [5] that any separable Banach space containing $\left(c_{0}\right)$ [resp. $\left.(c)\right]$ as a subspace may be projected onto $\left(c_{0}\right)$ [resp. $\left.(c)\right]$ by a projection $P$ with $\|P\| \leqq 2$ [resp. $\|P\| \leqq 3]$. Our methods are related to those of Sobczyk and McWilliams. (The author is indebted to Dr. R. D. McWilliams for an exposition of results of [5] and the corresponding proofs prior to their publication.) Related questions have been treated also by Dean [3].

We find it convenient to define, for a (real, or complex) Banach space $X$ and a (finite, or infinite) cardinal $k$, the projection constant $P_{k}^{\prime}(X)$ as follows:

$P_{\boldsymbol{k}}(X)$ is the greatest lower bound of reals $p$ which have the property: If $Y$ is a Banach space, if $X \subset Y$, and if the quotient-space $Y / X$ contains a dense set of cardinality less than or equal to $k$, then there exists $a$ projection $P$ of $Y$ onto $X$ such that $\|P\| \leqq p$.

If $\boldsymbol{P}_{\boldsymbol{k}}(X)$ is not only the greatest lower bound but the minimum of the numbers $p$, we call the projection constant exact.

The interest in the projection constants stems at least in part from their connection with extensions of linear transformations, which may be formulated as follows:

$P_{k}(X)$ is the greatest lower bound of reals $p$ having the property: For any Banach spaces $Y$ and $Z$, where $Y \subset Z$ and $Z / Y$ contains a dense set of cardinality less than or equal to $k$, and for any linear transformation $f$ of $Y$ into $X$, there exists a linear transformation $F$ of $Z$ into $X$, coinciding with $f$ on $Y$ and such that $\|F\| \leqq p \cdot\|f\|$.

This characterization of $P_{k}(X)$ may be easily established either by using a theorem on extensions of linear transformations due to Sobczyk [9, Theorem 4.1], or by observing the following well-known facts:

(i) Any Banach space may be isometrically imbedded in the space

Presented to the Society, November 3, 1959; received by the editors November 9 , 1959.

1 Paper written under contract Nonr 58304 with Office of Naval Research at the University of Kansas, Lawrence, Kansas. 
$m(A)$ of all bounded functions defined on a suitable set $A[7$, p. 538 , footnote 12].

(ii) Any $m(A)$ space has the Hahn-Banach extension property [6; 7, Corollary 7.2]; in particular, $\boldsymbol{P}_{\boldsymbol{k}}(m(A))=1$ and is exact for any cardinal $k$.

(Statements related to the above have been proved by various authors; references may be found in Day [2, p. 947].)

The following notation will be used throughout the sequel:

$A=\{\alpha\}$ denotes a set of cardinality card $A=$ a. $m(A)$ [resp. $\tilde{m}(A)$ ] is the set of all bounded real [complex] valued functions $x=(x(\alpha), \alpha \in A)$, considered as a real [complex] Banach space with norm $\|x\|=\sup _{\alpha \in A}|x(\alpha)|$.

For any $\epsilon \geqq 0$ and any $x$ belonging to $m(A)$ or $\tilde{m}(A)$ let $N(x ; \epsilon)$ denote the cardinal number of the subset $A(x ; \epsilon)$ of $A$ defined by $A(x, \epsilon)=\{\alpha \in A ;|x(\alpha)|>\epsilon\}$.

The following statements are easily verified:

(1) If $0 \leqq b<a$, then the subset $\{x ; N(x ; 0) \leqq b\}=\{x ; N(x ; \epsilon) \leqq b$ for each $\epsilon>0\}$ of $m(A)$ [resp. $\tilde{m}(A)]$ is a Banach space. We shall denote it by $c_{b}(A)\left[\right.$ resp. $\left.\tilde{c}_{b}(A)\right]$.

(2) If $\boldsymbol{a} \geqq \boldsymbol{\aleph}_{0}$, then the subset $\left\{x ; N(x, \epsilon)<\boldsymbol{\aleph}_{0}\right.$ for each $\left.\epsilon>0\right\}$ of $m(A)[\operatorname{resp} . \tilde{m}(A)]$ is a Banach space. We shall denote it by $c_{0}(A)$ $\left[\right.$ resp. $\left.\tilde{\varepsilon}_{0}(A)\right]$.

With this notation we may formulate our results as follows:

TheOREM 1. If $\boldsymbol{a} \geqq \boldsymbol{N}_{0}$ then $\boldsymbol{P}_{\boldsymbol{N}_{0}}\left(c_{0}(A)\right)=\boldsymbol{P}_{\boldsymbol{N}_{0}}\left(\tilde{\varepsilon}_{0}(A)\right)=2$; if $\boldsymbol{a}>\boldsymbol{b} \geqq \boldsymbol{N}_{0}$ then $\boldsymbol{P}_{b}\left(c_{b}(A)\right)=\boldsymbol{P}_{b}\left(\tilde{\tau}_{b}(A)\right)=2$. Moreover, all these projection constants are exact.

THEOREM 2. If $X$ is a subspace of $m(A)$ and contains $c_{0}(A)$ [resp. $\left.c_{\beta}(A)\right]$ as a subspace of deficiency 1 , then

$$
P_{\mathbb{N}_{0}}(X)=3\left[\text { resp. } P_{b}(X)=3\right]
$$

and is exact. The same result holds in the complex case.

THEOREM 3. If $X$ is a subspace of $m(A)$ and contains $c_{0}(A)$ [resp. $\left.c_{\beta}(A)\right]$ as a subspace of deficiency $n$, then

$$
P_{\aleph_{0}}(X) \leqq n+2\left[\text { resp. } P_{b}(X) \leqq n+2\right] .
$$

The proof of Theorem 1 will be given in $\S 3$, after we establish in $\$ 2$ a simple lemma of a combinatorial character, which is crucial in our proofs. Theorems 2 and 3 are proved in $\$ 4$, while $\$ 5$ contains some remarks and examples. 
2. A combinatorial lemma. Let $M=\left\{M_{\beta}^{\alpha}\right\}$ denote an array whose rows are indexed by ordinals $\beta$ with $1 \leqq \beta<\Omega$, where $\Omega$ is the initial ordinal of an infinite cardinal $\leqq b$, and whose columns have indices $\alpha$ belonging to a set $A$ of cardinality card $A=a$. The elements of $\boldsymbol{M}$ are infinite sequences $M_{\beta}^{\alpha}=\left(\phi_{\beta}^{\alpha}(n), 1 \leqq n<\infty\right)$ where each $\phi_{\beta}^{\alpha}(n)$ is one of the symbols 0 and 1 . For any fixed $k, 1 \leqq k<\infty$, and any fixed $\beta_{0}, 1<\beta_{0}<\Omega$, let $\boldsymbol{M}\left(k, \beta_{0}\right)$ denote the array $\boldsymbol{M}\left(k, \beta_{0}\right)=\left\{M_{\beta}^{\alpha}(k)\right\}$, $1 \leqq \beta<\beta_{0}, \alpha \in A$, whose elements are the initial segments of length $k$ of the corresponding $M_{\beta}^{\alpha}$, i.e. $M_{\beta}^{\alpha}(k)=\left(\phi_{\beta}^{\alpha}(1),\left(\phi_{\beta}^{\alpha}(2), \cdots, \phi_{\beta}^{\alpha}(k)\right)\right)$.

Lemma. Given any array $\boldsymbol{M}=\left\{M_{\beta}^{\alpha}\right\}$ of the type described, there exists an array $\tilde{M}=\left\{\tilde{M}_{\beta}^{\alpha}\right\}$ of the same type which satisfies the following conditions:

(i) If $\boldsymbol{\aleph}_{0} \leqq b<a$, then for each $n$ and each $\beta$ the inequality $\phi_{\beta}^{\alpha}(n)$ $\neq \tilde{\phi}_{\beta}^{\alpha}(n)$ takes place for at most $b$ different $\alpha \in A$, while for each $k$ and each $\beta_{0}$ every column of $\tilde{\boldsymbol{M}}\left(k, \beta_{0}\right)$ occurs more than $\boldsymbol{b}$ times.

(ii) If $\boldsymbol{\aleph}_{0}=b \leqq a$, then for each $n$ and each $\beta$ the inequality $\phi_{\beta}^{\alpha}(n)$ $\neq \bar{\phi}_{\beta}^{\alpha}(n)$ takes place for at most a finite number of $\alpha \in A$ while for each $k$ and each $\beta_{0}$ every column of $\tilde{M}\left(k, \beta_{0}\right)$ occurs infinitely many times.

Proof. We first consider the assertion (i). We shall prove it by defining $\tilde{\phi}_{\beta}^{\alpha}(n)$ in an appropriate way, using transfinite induction on $\beta$ and induction on $n$.

For $\beta=1$ we consider the set $\left\{\phi_{1}^{\alpha}(1) ; \alpha \in A\right\}$. Since card $A=a>b$, at least one of the equations $\phi_{1}^{\alpha}(1)=0, \phi_{1}^{\alpha}(1)=1$ holds for more than $b$ indices $\alpha \in A$. If both are fulfilled for more than $b$ different indices, we define $\tilde{\phi}_{1}^{\alpha}(1)=\phi_{1}^{\alpha}(1)$ for all $\alpha \in A$. If only one of them holds more than $b$ times, let us say the first, we define $\tilde{\phi}_{1}^{\alpha}(1)=0$ for all $\alpha \in A$. Obviously, $\phi_{1}^{\alpha}(1) \neq \phi_{1}^{\alpha}(1)$ for at most $b$ different $\alpha \in A$.

Assuming $\tilde{\phi}_{1}^{\alpha}(k)$ already defined for $1 \leqq k \leqq n$ and $\alpha \in A$, we define $\tilde{\phi}_{1}^{\alpha}(n+1)$ as follows. We consider the ordered $n$-tuples $\left(\tilde{\phi}_{1}^{\alpha}(1), \tilde{\phi}_{1}^{\alpha}(2), \cdots, \tilde{\phi}_{1}^{\alpha}(n)\right)$. The set $A$ may be partitioned into $N \leqq 2^{n}$ disjoint subsets $A_{i}$ with $\bigcup_{i=1}^{N} A_{i}=A$, such that card $A_{i}>b$ for each $i$ and, if $\alpha, \alpha^{*} \in A_{i}$ then $\tilde{\phi}_{1}^{\alpha}(k)=\tilde{\phi}_{1}^{\alpha *}(k)$ for all $k, 1 \leqq k \leqq n$. For each $i$, either $\phi_{1}^{\alpha}(n+1)=0$, or $\phi_{1}^{\alpha}(n+1)=1$, or both, occur for more than $b$ indices $\alpha \in A_{i}$. Therefore, as above, we may define $\tilde{\phi}_{1}^{\alpha}(n+1)$ properly for all $\alpha \in A$ by taking $\tilde{\phi}_{1}^{\alpha}(n+1) \neq \phi_{1}^{\alpha}(n+1)$ for at most $N \cdot b=b$ indices $\alpha \in A$. Thus the step $\beta=1$ of the transfinite induction on $\beta$ is completed.

Assuming $\tilde{\phi}_{\beta}^{\alpha}(k)$ defined for all $\beta<\beta_{0}$ for some $\beta_{0}$ with $1<\beta_{0}<\Omega$, $1 \leqq k<\infty$ and $\alpha \in A$, as well as for $\beta=\beta_{0}, k<n$ with $n \geqq 1$, and $\alpha \in A$, we consider, in analogy to the above, the arrays 


$$
\Phi^{\alpha}=\left\{\begin{array}{ccccc}
\tilde{\phi}_{1}^{\alpha}(1) & \tilde{\phi}_{1}^{\alpha}(2) & \cdots & \tilde{\phi}_{1}^{\alpha}(n-1) & \tilde{\phi}_{1}^{\alpha}(n) \\
\tilde{\phi}_{2}^{\alpha}(1) & \tilde{\phi}_{2}^{\alpha}(2) & \cdots & \tilde{\phi}_{2}^{\alpha}(n-1) & \tilde{\phi}_{2}^{\alpha}(n) \\
\vdots & \vdots & & \vdots & \vdots \\
\tilde{\phi}_{\beta}^{\alpha}(1) & \tilde{\phi}_{\beta}^{\alpha}(2) & \cdots & \tilde{\phi}_{\beta}^{\alpha}(n-1) & \tilde{\phi}_{\beta}^{\alpha}(n) \\
\vdots & \vdots & & \vdots & \vdots \\
\tilde{\phi}_{\beta_{0}}^{\alpha}(1) & \tilde{\phi}_{\beta_{0}}^{\alpha}(2) & \cdots & \tilde{\phi}_{\beta_{0}}^{\alpha}(n-1) & 0
\end{array}\right\}
$$

for $\alpha \in A$. Using the inductive hypothesis, it is possible to partition $A$ into disjoint subsets $A_{i}, i \in I$, where card $I \leqq 2^{\text {card } \beta_{0}} \leqq b$ in such a way that card $A_{i}>b$ for each $i \in I$ and that $\alpha, \alpha^{*} \in A_{i}$ imply $\Phi^{\alpha}=\Phi^{\alpha *}$. Again, for each $i \in I$, either $\phi_{\beta_{0}}^{\alpha}(n)=0$, or $\phi_{\beta_{0}}^{\alpha}(n)=1$, or both, hold for more than $b$ indices $\alpha \in A$. Therefore, by taking $\tilde{\phi}_{\beta_{0}}^{\alpha}(n) \neq \phi_{\beta_{0}}^{\alpha}(n)$ for at most card $I \cdot b \leqq b^{2}=b$ different $\alpha \in A$, we may define $\tilde{\phi}_{\beta_{0}}^{\alpha}(n)$ in the proper way for all $\alpha \in A$.

By the construction of $\tilde{M}$ it is evident that for each $k$ and each $\beta_{0}<\Omega$ every column of $\tilde{M}\left(k, \beta_{0}\right)$ is repeated for more than $b$ different $\alpha \in A$, which proves assertion (i) of the lemma.

In order to prove (ii), we have only to observe that it is possible to repeat the above argument substituting "finitely many" for "not more than $b$," and "infinitely many" for "more than $b$." This ends the proof of the lemma.

3. Proof of Theorem 1. We begin with the proof of the statements concerning $P_{b}\left(c_{b}(A)\right)$. If $Y$ is any Banach space containing $c_{b}(A)$ there exists a linear transformation $T$ of $Y$ into $m(A)$ such that $T(x)=x$ for any $x \in c_{b}(A)$, and $\|T\|=1$ [7, Corollary 7.2]. Then, if $P_{0}$ is any projection of $T(Y)$ onto $c_{b}(A)$ with $\left\|P_{0}\right\| \leqq 2$, we obtain a projection of $P$ of $Y$ onto $c_{b}(A)$ by defining $P=P_{0} T$. Since $\|P\|$ $\leqq\left\|P_{0}\right\| \cdot\|T\| \leqq 2$ we shall establish $P_{b}\left(c_{b}(A)\right) \leqq 2$ by constructing, for any $Y$ with $c_{b}(A) \subset Y \subset m(A)$ such that $Y / c_{b}(A)$ has a dense subset of cardinality at most $b$, a projection $P$ of $Y$ onto $c_{b}(A)$ with $\|P\| \leqq 2$. (The same reduction is possible in all the proofs of the present paper; we shall therefore in the sequel always assume $Y \subset m(A)$ [resp. $Y \subset \tilde{m}(A)]$.)

If $Y \subset m(A)$ let $D$ be a subset of $Y$, with card $D \leqq b$, such that $Y$ is the closed linear hull of $D \cup c_{b}(A)$. Let $\Omega$ denote the initial ordinal of the cardinal card $D$, and let $\left\{y_{\beta}, 1 \leqq \beta<\Omega\right\}$ be a well-ordering of $D$. Without loss of generality we assume, moreover, that $D$ satisfies: 
(i) $\left\|y_{\beta}\right\|=1$ for each $\beta$;

(ii) for each $\beta$ and each $\epsilon>0$ we have $N\left(y_{\beta}, 1-\epsilon\right)>b$ (see §2);

(iii) any finite subfamily of $D$ is linearly independent over $c_{b}(A)$.

For $x \in m(A)$ we define \|\|$x \|_{b}=\inf \left\{\left\|x+x^{\prime}\right\|: x^{\prime} \in c_{b}(A)\right\}$. Obviously, $\|x \mid\|_{b} \leqq\|x\|$ for all $x \in m(A)$. Properties (i) and (ii) may then be expressed as $\left\|y_{\beta}\right\|_{b}=1$.

Now, for each $\alpha \in A$ and $\beta<\Omega$, let $0 \cdot y_{\beta}^{1}(\alpha) y_{\beta}^{2}(\alpha) \cdots y_{\beta}^{n}(\alpha) \cdots$ be a representation of $\left|y_{\beta}(\alpha)\right|$ as a dyadic fraction, i.e. each $y_{\beta}^{n}(\alpha)$ is either 0 or 1 . We define an array $M$ of the kind discussed in $\$ 2$ by putting

$$
\begin{array}{lr}
\underset{\phi_{\beta}^{\alpha}(1)}{\alpha}=\left\{\begin{array}{lr}
0 & \text { if } y_{\beta}(\alpha)<0, \\
1 & \text { if } y_{\beta}(\alpha) \geqq 0,
\end{array}\right. \\
\phi_{\beta}^{\alpha}(n)=y_{\beta}^{n-1}(\alpha) & \text { for } n>1,
\end{array}
$$

for all $\alpha \in A, \beta<\Omega$.

Let $\tilde{M}$ be the array whose existence was established in the lemma of $\S 2$, obtained from $M$ by the construction used in the proof of the lemma. Let $\tilde{D}=\left\{\tilde{y}_{\beta}, \beta<\Omega\right\}$ be the subset of $m(A)$ obtained from $\tilde{M}$ by the above correspondence. The properties of $\tilde{M}$ and the method of its construction, together with (i), (ii), and (iii), imply that:

(1) $\left\|\tilde{y}_{\beta}\right\|=\left\|\tilde{y}_{\beta}\right\| \|_{b}=1$ for each $\beta<\Omega$;

(2) $\tilde{y}_{\beta}-y_{\beta} \in c_{b}(A)$ for each $\beta<\Omega$; this implies that the linear hull of $\tilde{D} \cup c_{b}(A)$ coincides with that of $D \cup c_{b}(A)$, and therefore $Y$ is the closed linear hull of $\tilde{D} \cup c_{b}(A)$.

(3) $\tilde{D}$ is linearly independent over $c_{b}(A)$, and therefore the linear hull of $\tilde{D}$ has only the origin in common with $c_{b}(A)$.

The advantage of $\tilde{D}$ over $D$ is

(4) If $B$ is any finite set of ordinals $<\Omega$, then for any $x \in c_{b}(A)$ and reals $\lambda_{\beta}, \beta \in B$, we have $\left\|x+\sum_{\beta \in B} \lambda_{\beta} \tilde{y}_{\beta}\right\| \geqq\left\|\sum_{\beta \in B} \lambda_{\beta} \tilde{y}_{\beta}\right\|$.

To establish (4) it is sufficient to remark that, as a consequence of (1), we have $\left\|\sum_{\beta \in B} \lambda_{\beta} \tilde{y}_{\beta}\right\|=\left\|\mid \sum_{\beta \in B} \lambda_{\beta} \tilde{y}_{\beta}\right\|_{b}=\left\|x+\sum_{\beta \in B} \lambda_{\beta} \tilde{y}_{\beta}\right\|_{b}$ $\leqq\left\|x+\sum_{\beta \in B} \lambda_{\beta} \tilde{y}_{\beta}\right\|$.

Now we define a projection $P_{0}$ from the linear hull of $c_{b}(A) \cup \tilde{D}$ onto $c_{b}(A)$ by putting $P_{0}(x)=x$ for $x \in c_{b}(A)$ and $P_{0}\left(\tilde{y}_{\beta}\right)=0$ for $\beta<\Omega$.

Then, using (4), we have $\left\|P_{0}\left(x+\sum_{\beta \in B} \lambda_{\beta} \tilde{y}_{\beta}\right)\right\|=\left\|P_{0}(x)\right\|=\|x\|$ $\leqq\left\|x+\sum_{\beta \in B} \lambda_{\beta} \tilde{y}_{\beta}\right\|+\left\|\sum_{\beta \in B} \lambda_{\beta} \tilde{y}_{\beta}\right\| \leqq 2 \cdot\left\|x+\sum_{\beta \in B} \lambda_{\beta} \tilde{y}_{\beta}\right\|$ i.e. $\left\|P_{0}\right\| \leqq 2$.

Extending $P_{0}$ by continuity we obtain the desired projection $P$ of $Y$ onto $c_{b}(A)$ with $\|P\| \leqq 2$.

On the other hand, it is easily seen that $P_{b}\left(c_{b}(A)\right) \geqq 2$ (in fact, $\left.P_{1}\left(c_{b}(A)\right) \geqq 2\right)$. Therefore, as claimed, $P_{b}\left(c_{b}(A)\right)=2$ and is exact.

In order to prove Theorem 1 for spaces $c_{0}(A)$ the same arguments 
may be applied, except for using the second part of the lemma of $\$ 2$ instead of its first part, and replacing (ii) by

(ii*) for each $\beta$ we have $\left|y_{\beta}(\alpha)\right|=1$ for infinitely many $\alpha \in A$. We shall express (i) and (ii*) by $\left\|\left|y_{\beta}\right|\right\|=1$.

The same method applies also to the complex spaces $\tau_{b}(A)$ and $\tilde{c}_{0}(A)$, the only difference being in the way of associating the array $M$ to the set $D$ (and $\tilde{D}$ to $\tilde{M}$ ), which is done as follows.

For $\alpha \in A$ and $\beta<\Omega$, let $y_{\beta}(\alpha)=\left|y_{\beta}(\alpha)\right| \cdot e^{2 \pi i \psi_{\beta}(\alpha)}$, with $0 \leqq \psi_{\beta}(\alpha)<1$, and let $\left|y_{\beta}(\alpha)\right|=0 \cdot y_{\beta}^{1}(\alpha) y_{\beta}^{2}(\alpha) \cdots y_{\beta}^{n}(\alpha) \cdots$ and $\psi_{\beta}(\alpha)=0$ $\cdot \psi_{\beta}^{1}(\alpha) \psi_{\beta}^{2}(\alpha) \cdots \psi_{\beta}^{n}(\alpha) \cdots$ by the dyadic representations of $y_{\beta}(\alpha)$ and $\psi_{\beta}(\alpha)$. Then we define $\phi_{\beta}^{\alpha}(2 n-1)=y_{\beta}^{n}(\alpha)$ and $\phi_{\beta}^{\alpha}(2 n)=\psi_{\beta}^{n}(\alpha)$ and the same correspondence is used for obtaining $\tilde{D}$ from $\tilde{M}$.

This ends the proof of Theorem 1.

4. Proof of Theorems 2 and 3. We start with the following observation. Let $Y \supset X \supset X_{0}$, where $X_{0}$ is a subspace of $X$ of deficiency 1 , and let $P_{0}$ be a projection of $Y$ onto $X_{0}$. If $x^{*} \in X$ but $x^{*} \notin X_{0}$, each element $x \in X$ has a unique representation in the form $x=\lambda x^{*}+x_{0}$, with $x_{0} \in X_{0}$. Let the functional $f_{x^{*}}$ on $X$ be defined by $f_{x^{*}}(x)$ $=f_{x^{*}}\left(\lambda x^{*}+x_{0}\right)=\lambda$, and let $F_{x^{*}}$ be any extension of $f_{x^{*}}$ to $Y$, with $\left\|F_{x^{*}}\right\|=\left\|f_{x^{*}}\right\|$. Then it is easily seen that the transformation $P(y)$ $=P_{0}(y)+F_{x^{*}}(y)\left(x^{*}-P_{0}\left(x^{*}\right)\right)$ is a projection of $Y$ onto $X$ with $\|P\|$ $\leqq\left\|P_{0}\right\|+\left\|F_{x^{*}}\right\| \cdot\left\|x^{*}-P_{0}\left(x^{*}\right)\right\|$.

In order to apply this remark to the proof of Theorem 2, let $X$ satisfy the conditions of Theorem 2 . We take a subset $D$ of $Y$, $X \subset Y \subset m(A)$, such that: (i) card $D \leqq \aleph_{0}$ [resp. card $\left.D \leqq b\right]$; (ii) $Y$ is the closed linear hull of $D \cup c_{0}(A)$ [resp. $D \cup c_{b}(A)$ ]; (iii) $y_{1} \in X$; (iv) the conditions (i), (ii*) and (iii) [resp. (i), (ii), and (iii)] of $\$ 3$ are satisfied. As in the proof of Theorem 1 , from $D$ we obtain another subset $\tilde{D}$ of $Y$, and a projection $P_{0}$ of $Y$ onto $c_{0}(A)$ [resp. $\left.c_{b}(A)\right]$ such that $\left\|P_{0}\right\| \leqq 2$ and $P_{0}\left(\tilde{y}_{\beta}\right)=0$ for all $\tilde{y}_{\beta} \in \tilde{D}$. On the other hand, property (2) of $\tilde{D}(\S 3)$ implies that $\tilde{y}_{1} \in X$, and since $\left\|\lambda \tilde{y}_{1}\right\|=\|\| \tilde{y}_{1} \|$ $=\|\| \lambda \tilde{y}_{1}+x_{0}\|\| \leqq\left\|\lambda \tilde{y}_{1}+x_{0}\right\|$ [resp. $\left\|\lambda y_{1}\right\|=\|\| \lambda y_{1}\left|\left\|_{b}=\right\|\right|\left\|\lambda y_{1}+x_{0}\right\|_{b}$ $\left.\leqq\left\|\lambda y_{1}+x_{0}\right\|\right]$ for any $x_{0} \in c_{0}(A)\left[\operatorname{resp} . x_{0} \in c_{b}(A)\right]$ we have $\left\|f_{\tilde{y}_{1}}\right\|=1$. Therefore, taking $x^{*}=\tilde{y}_{1}$ we obtain a projection $P$ of $Y$ onto $X$ with $\|P\| \leqq\left\|P_{0}\right\|+\left\|F_{\tilde{y}_{1}}\right\| \cdot\left\|\tilde{y}_{1}\right\| \leqq 3$.

Obviously, the same reasoning applies to the complex case. Thus, in order to complete the proof of Theorem 2 we have to show that $P_{\aleph_{0}}(X) \geqq 3$, and similarly for the other projection constants. Examples to that effect have been given by McWilliams for the space $(c)$ of all converging sequences; his example may readily be adapted to any space $X$ of the type considered in Theorem 2 .

This ends the proof of Theorem 2 . 
It may be remarked that on applying the reasoning given at the beginning of $\$ 4$, Theorem 1 yields the following result: If $X$ is any space containing $c_{0}(A)$ as a subspace of deficiency 1 then $P_{\aleph_{0}}(X) \leqq 5$; similarly for $X$ containing $c_{b}(A), \tilde{c}_{0}(A)$ or $\tilde{c}_{b}(A)$.

In order to prove Theorem 3, we take any $Y, X \subset Y \subset m(A)$, which is the closed linear hull of $D \cup c_{0}(A)$ [resp. $D \cup_{c_{b}}(A)$ ], where $D$ satisfies: (i) card $D \leqq \aleph_{0}$ [resp. card $\left.D \leqq b\right]$; (ii) conditions (i), (ii*) [resp. (ii)] and (iii) of $\S 3$; (iii) $y_{\beta} \in X$ for $1 \leqq \beta \leqq n$.

As in $\S 3$, we obtain a set $\widetilde{D}=\left\{\tilde{y}_{\beta}\right\}$, and a projection $P_{0}$ of $Y$ onto $c_{0}(A)$ [resp. $\left.c_{b}(A)\right]$ such that $\left\|P_{0}\right\| \leqq 2, P_{0}(x)=x$ for $x \in c_{0}(A)$ [resp. $\left.x \in c_{b}(A)\right]$ and $P_{0}\left(\tilde{y}_{\beta}\right)=0$ for all $\tilde{y}_{\beta} \in \tilde{D}$. On the other hand, by (2) of $\S 3$, we have $\tilde{y}_{\beta} \in X$ for $1 \leqq \beta \leqq n$.

Now we apply to the $n$-dimensional space $Z \subset X$ spanned by the $n$ independent elements $\tilde{y}_{\beta}, 1 \leqq \beta \leqq n$, the following result due to Taylor [10] and Day [1]:

In any $n$-dimensional real space $Z$ there exist elements $z_{i}, 1 \leqq i \leqq n$, and functionals $f_{i}, 1 \leqq i \leqq n$, such that $\left\|z_{i}\right\|=\left\|f_{i}\right\|=1$ for $1 \leqq i \leqq n$ and $f_{i}\left(z_{k}\right)=\delta_{k}^{i}$.

On $X=c_{0}(A) \oplus Z$ [resp. $X=c_{b}(A) \oplus Z$ ] we define functionals $f_{i}^{*}$ by $f_{i}^{*}(x+z)=f_{i}(z)$ for $1 \leqq i \leqq n$, where $x \in c_{0}(A) \quad\left[\right.$ resp. $\left.x \in c_{b}(A)\right]$ and $z \in Z$.

Since the points $z_{i}$ are linear combinations of the points $y_{\beta}$ with $1 \leqq \beta \leqq n$, and $\left\|y_{\beta} \mid\right\|=1$ [resp. $\left\|y_{\beta}\right\|_{b}=1$ ], we have $\left\|f_{i}^{*}\right\|=1$ for $1 \leqq i \leqq n$. On the other hand, obviously $P_{0}\left(z_{i}\right)=0$ for $1 \leqq i \leqq n$. Let now $F_{i}$ denote an extension of $f_{i}^{*}$ to $Y$, with $\left\|F_{i}\right\|=1,1 \leqq i \leqq n$. Then $P(y)=P_{0}(y)+\sum_{i=1}^{n} F_{i}(y) z_{i}$ is a projection of $Y$ onto $X$, and clearly $\|P\| \leqq\left\|P_{0}\right\|+\sum_{i=1}^{n}\left\|F_{i}\right\| \cdot\left\|z_{i}\right\| \leqq 2+n$.

This ends the proof of Theorem 3 .

5. Remarks. It would be interesting to know whether Theorem 2 remains true if the condition $X \subset m(A)$ is removed. The method used in $\$ 4$ seems not to be applicable to this case. Indeed, there exist spaces $X$ containing e.g. $\left(c_{0}\right)$ as a subspace of deficiency 1 such that for any $x \in X$ there exists $x_{0} \in\left(c_{0}\right)$ with $\left\|x+x_{0}\right\|<\|x\|$, i.e. no $x^{*} \in X$ satisfies $\left\|x^{*}\right\|=\left\|f_{x^{*}}\right\|=1$. An easy example of such a space $X$ is the space of all sequences $x=\left(x^{(n)}, 0 \leqq n<\infty\right)$ with $\lim _{n \rightarrow \infty} x^{(n)}=0$ and norm

$$
\|x\|=\sup _{1 \leqq n<\infty}\left\{\left|x^{(n)}\right|,\left|x^{(0)}+\frac{1}{n}\left(x^{(0)}+x^{(n)}\right)\right|\right\} .
$$

Obviously, this example as well as the following one may be suitably modified for any $c_{0}(A)$ or $c_{b}(A)$ space.

We do not know whether the bound $n+2$ in Theorem 3 is the best 
possible. The following construction shows that for $X$ containing $\left(c_{0}\right)$ as a subspace of deficiency $n$, we may have

$$
P_{\aleph_{0}}(X) \geqq v_{n}=\frac{n}{2^{n-1}} \cdot\left(\left[\begin{array}{c}
n-1 \\
{\left[\frac{n-1}{2}\right]}
\end{array}\right] \sim\left(\frac{2 n}{\pi}\right)^{1 / 2} .\right.
$$

Let $n$ be given and let $N=2^{n-1}$. In [4] it was proved that there exists an $N$-dimensional space $Z_{N}$ and an $n$-dimensional subspace $X_{n}$ of $Z_{N}$ such that:

(i) Any projection $P$ of $Z_{N}$ onto $X_{n}$ satisfies $\|P\| \geqq v_{n}$.

(ii) There exists a basis $\left\{z_{i}\right\}_{i=1}^{N}$ of $Z_{N}$ which may be represented by sequences $z_{i}=\left(z_{i}^{(k)}\right), 1 \leqq k \leqq N$, where each $z_{i}^{(k)}$ is either +1 or -1 , with the properties: (1) $\left\{z_{i}\right\}_{i=1}^{n}$ is a basis for $X_{n}$; (2) $\left\|\sum_{i=1}^{N} \lambda_{i} z_{i}\right\|$ $=\max _{1 \leq k \leq N}\left|\sum_{i=1}^{N} \lambda_{i} z_{i}^{(k)}\right|$.

Now let $Y$ be the subspace of $(m)$ spanned by $\left(c_{0}\right)$ and the sequences $y_{i}=\left(y_{i}^{(k)}\right), \quad 1 \leqq k<\infty, 1 \leqq i \leqq N$, where $y_{i}^{(k)}=z_{i}^{(j)}$ for $1 \leqq i \leqq N$ and $1 \leqq j \leqq N, j \equiv k(\bmod N)$. If $Y_{0} \subset Y$ is the linear hull of $\left\{y_{i}\right\}_{i=1}^{N}$, and $X_{0}$ that of $\left\{y_{i}\right\}_{i=1}^{n}$, it is obvious that $Y_{0}$ is equivalent to $Z_{N}$ and $X_{0}$ to $X_{n}$.

Denoting $X=\left(c_{0}\right) \oplus X_{0}$, let $P$ be any given projection of $Y$ onto $X$. Then, for $n<i \leqq N$ we have $P\left(y_{i}\right)=x_{i}+\sum_{k=1}^{n} \lambda_{i}^{(k)} y_{k}$, where $x_{i} \in\left(c_{0}\right)$. Defining $P^{*}\left(y_{i}\right)=\sum_{k=1}^{n} \lambda_{i}^{(k)} y_{k}$, for $n<i \leqq N$, we obtain a projection of $Y_{0}$ onto $X_{0}$. Since

$$
\begin{aligned}
\left\|\sum_{i=1}^{n} \lambda_{i}^{(k)} y_{k}\right\| & =\|\| \sum_{i=1}^{n} \lambda_{i}^{(k)} y_{k}\|\|=\left\|x_{i}+\sum_{i=1}^{n} \lambda_{i}^{(k)} y_{k}\right\| \| \\
& \leqq\left\|x_{i}+\sum_{i=1}^{n} \lambda_{i}^{(k)} y_{k}\right\|
\end{aligned}
$$

it follows that $\left\|P^{*}\right\| \leqq\|P\|$ and therefore, by the equivalence of $Y_{0}, X_{0}$ with $Y_{N}, X_{n}$, we have $\|P\| \geqq v_{n}$ as cläimed.

\section{REFERENCES}

1. M. M. Day, Polygons circumscribed about closed convex curves, Trans. Amer. Math. Soc. 62 (1947), 315-319.

2. - Normed linear spaces, Springer, Berlin, 1958.

3. D. W. Dean, Projections in certain continuous function spaces, Thesis, University of Illinois, 1958. 465.

4. B. Grünbaum, Projection constants, Trans. Amer. Math. Soc. 95 (1960), 451-

5. R. D. McWilliams, On projections of separable subspaces of $(m)$ onto $(c)$, Proc. Amer. Math. Soc. 10 (1959), 872-876. 
6. L. Nachbin, A theorem of the Hahn-Banach type for linear transformations, Trans. Amer. Math. Soc. 68 (1950), 28-46.

7. R. S. Phillips, On linear transformations, Trans. Amer. Math. Soc. 48 (1940), 516-541.

8. A. Sobczyk, Projections of the space ( $m)$ on its subspace $\left(c_{0}\right)$. Bull. Amer. Math. Soc. 47 (1941), 938-947.

9. - On the extension of linear transformations, Trans. Amer. Math. Soc. 55 (1944), 153-169.

10. A. E. Taylor, $A$ geometric theorem and its application to biorthogonal systems, Bull. Amer. Math. Soc. 53 (1947), 614-616.

INSTITUTE FOR ADVANCED STUdY

\section{DENSE ALGEBRAS OF FUNCTIONS IN $L_{p}$}

\section{R. H. FARRELL 1}

1. Introduction. Several of the known examples of sets of functions dense in $L_{2}(0,1)$ are in fact dense algebras of functions. For example the Walsh functions are closed under pointwise multiplication and therefore the linear combinations of these functions form an algebra. The linear space generated by $1,\{\sin (n \theta), n \geqq 1\},\{\cos (n \theta), n \geqq 1\}$ is again an algebra. It is suggested then that perhaps the conditions of the Stone-Weierstrass theorem can be given a measure-theoretic interpretation which would allow proof of a corresponding theorem in the $L_{p}$ spaces. It is the purpose of this paper to state and prove such a theorem. We will assume that a locally-compact topological space $X$ is given and $B$ is the class of Baire sets of $X$. We assume $B$ is a $\sigma$-algebra containing $X$; therefore that $X$ is a Baire set.

We prove the following theorem.

THEOREM. Let $\mu$ be a real valued $\sigma$-finite Baire measure on $X, \bigotimes$. Let $E$ be an algebra of real valued essentially bounded Baire measurable functions. Suppose $1 \leqq p<\infty$ is given and $E \subset L_{p}(\mu)$. Suppose

(1) there is an $h \in E$ such that $h>0$ a.e. $\mu$;

(2) there is given a base $\left\{U_{\alpha}\right\}$ for the open Baire sets of $X$ such that if $U_{\alpha} \cap U_{\beta}=\Phi$ then for some $h \in E, h \leqq 0$ a.e. $\mu$ on $U_{\alpha}$ and $h>0$ a.e. $\mu$ on $U_{\beta}$. Then $E$ is dense in $L_{p}(\mu)$.

The proof is given in the next section. In the final section of this paper additional examples are given.

Received by the editors February 25, 1961.

1 Research sponsored by the Office of Naval Research under contract No. Nonr401(03). 